\title{
CUIDADO A PESSOAS COM TRANSTORNO MENTAL E FAMILIARES: DIAGNÓSTICOS E INTERVENÇÕES A PARTIR DA CONSULTA DE ENFERMAGEM
}

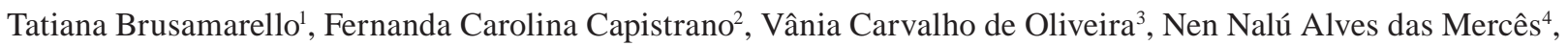
Mariluci Alves Maftum ${ }^{5}$

RESUMO: Estudo documental retrospectivo descritivo cujo objetivo foi descrever os diagnósticos eintervenções deenfermagem elaborados durante a consulta de enfermagem a pessoas com transtorno mental e familiares em um projeto de extensão universitária no ano de 2010. Foi utilizado um roteiro sistematizado que indicou maior frequência dos diagnósticos: enfretamento familiar comprometido, atividades de recreação deficientes, risco para vínculo pais e filhos prejudicado, interação social prejudicada, padrão de sono prejudicado, sofrimento moral, memória prejudicada, processo do pensamento perturbado, comportamento de saúde propenso a risco, nutrição desequilibrada e desesperança. As intervenções de enfermagem foram desenvolvidas de acordo com cada diagnóstico, direcionadas para a educação em saúde eapoio emocional. Concluiu-se pela relevância da aplicação de diagnósticos eintervenções de enfermagemà pessoas com transtornosmentais efamiliares, uma vez que estas intervenções proporcionam ao enfermeiro um cuidado direcionado, fundamentadona qualidadeena excelência. DESCRITORES: Enfermagem; Saúde mental; Processos de enfermagem.

\section{CARING PEOPLE WITH MENTAL DISORDERS AND THEIR FAMILY MEMBERS: DIAGNOSES AND INTERVENTIONS FROM NURSING CONSULTATION}

ABSTRACT: This descriptive, retrospective documental study aimed to describe the diagnoses and nursing interventions developed during the nursing consultation, for people with mental illnesses and their family members, in a university extension project in 2010. A systematized script was used which indicated a higher frequency of the following diagnoses: compromised family coping, insufficient recreational activities, risk for impaired link between parents and children, impaired social interaction, impaired sleep patterns, moral suffering, impaired memory, disturbed thought processes, risky health behaviors, unbalanced nutrition, and hopelessness. The nursing interventions were developed in line with each diagnosis, aimed at health education and emotional support. It was concluded that the application of nursing diagnoses and interventions for people with mental illnesses and their family members was relevant, because these interventions allow the nurse to provide directed care, based in quality and excellence. DESCRIPTORS: Nursing; Mental health; Nursing processes.

\section{CUIDADO A LAS PERSONAS CON TRASTORNOS MENTALES Y A LAS FAMILIAS: DIAGNÓSTICOS Y INTERVENCIONES A PARTIR DE LA CONSULTA DE ENFERMARÍA}

RESUMEN: Estudio documental retrospectivo descriptivo cuya finalidad fue describir los diagnósticos e intervenciones de enfermería creados durante la consulta de enfermería a personas con trastorno mental y familiares en un proyecto de extensión universitaria en el año de 2010. Fue utilizado un guión sistematizado que indicó mayor frecuencia de los diagnósticos: afrontamiento familiar comprometido, actividades de recreación deficientes, riesgo para vínculo entre padres e hijos perjudicado, interacción social perjudicada, patrón de sueño perjudicado, sufrimiento moral, memoria perjudicada, proceso del pensamiento perturbado, comportamiento de salud propenso a riesgo, nutrición desequilibrada y desesperanza. Las intervenciones de enfermería fueron desarrolladas de acuerdo con cada diagnóstico, condicionadas para la educación en salud y apoyo emocional. Se concluyó que es relevante la aplicación de diagnósticos e intervenciones de enfermería a personas con trastornos mentales y familiares, una vez que estas intervenciones proporcionan al enfermero un cuidado direccionado, fundamentado en la cualidad y en la excelencia. DESCRIPTORES: Enfermería; Salud mental; Procesos de enfermería.

\footnotetext{
${ }^{1}$ Enfermeira. Mestranda pelo Programa de Pós-Graduação em Enfermagem da Universidade Federal do Paraná-PPGENF UFPR. Bolsista CAPES. Membro do Núcleo de Estudos, Pesquisa e Extensão do Cuidado Humano de Enfermagem - NEPECHE.

${ }^{2}$ Acadêmica do Curso de Enfermagem da UFPR. Bolsista do Projeto de Extensão 'O Cuidado à saúde de pessoas com sofrimento mental e familiares'. Bolsista voluntária de Iniciação Científica. Membro do NEPECHE.

${ }^{3}$ Enfermeira. Mestranda pelo PPGENF UFPR. Membro do NEPECHE.

${ }^{4}$ Enfermeira. Doutora em Enfermagem. Professora do Curso de Graduação e do PPGENF UFPR. Membro do NEPECHE.

${ }^{5}$ Enfermeira. Doutora em Enfermagem. Professora do Curso de Graduação e do PPGENF UFPR. Vice-Coordenadora do NEPECHE. Coordenadora do PPGENF UFPR e do Projeto de Extensão 'O Cuidado à saúde de pessoas com sofrimento mental e familiares'.
} 


\section{INTRODUÇÃO}

Pessoas com transtornos mentais são uma parcela significativa da população que, muitas vezes, sofrem o preconceito, medo e exclusão da sociedade e dos próprios familiares. Contudo, as consequências do transtorno acometem tanto o indivíduo adoentado como a família e a comunidade, principalmente quando se trata do estado agudo da doença ${ }^{(1)}$.

A família está diretamente envolvida no processo de adoecer e cuidar de seus membros e desse modo, sofre as implicações da sobrecarga: emocionais, pela preocupação, impotência, irritação, medo e ansiedade; implicações físicas, como privação do sono, falta de apetite, problemas articulares e cardíacos; além das implicações econômicas ${ }^{(2-3)}$.

O movimento da Reforma Psiquiátrica Brasileira teve seu início no final dos anos 1970 e início de 1980. Desde então, esse tem proporcionado importantes contribuições no modo de cuidar em saúde mental e, com destaque, o estímulo à criação de uma rede de atenção à saúde extra-hospitalar ${ }^{(4)}$. Concomitantemente a isto, surgem novos espaços para atuação profissional do enfermeiro, como os Centros de Atenção Psicossocial (CAPS), Centros de Convivência, Serviços de Residência Terapêutica, Unidades Básicas de Saúde, entre outros, que resultam em possibilidades para o desenvolvimento do conhecimento e de diferentes habilidades no modo de cuidar da pessoa com transtorno mental ${ }^{(5)}$.

Além dos serviços que compõem a rede de atenção em saúde mental, a ideologia da Reforma Psiquiátrica é valorizar também a rede de apoio social formal e informal, como as associações, grupos de ajuda mútua e todo e qualquer espaço que venha agregar e contribuir no cuidado e na reinserção da pessoa com transtorno mental e que forneça apoio à família.

A prática profissional da enfermagem consiste, por excelência, no desenvolvimento e gerenciamento do cuidado ao ser humano. Destarte, o modo como ela se concretiza no cotidiano deve ser objeto de estudo e preocupação dos profissionais da enfermagem em uma permanente busca pela ampliação e solidificação do corpo de conhecimento específico, e aprimoramento técnico-científico, visando a um cuidado qualificado ${ }^{(6)}$.

Nesta perspectiva, a consulta de enfermagem possibilita a aproximação pessoa a pessoa, estabelecendo uma relação terapêutica individualizada. Trata-se de uma atividade assistencial sistematizada de aplicação do processo de enfermagem, compreendendo algumas etapas que direcionam as ações do enfermeiro, entre elas, o histórico, diagnóstico, plano assistencial, prescrição, evolução e prognóstico de enfermagem ${ }^{(7-8)}$.

A consulta de enfermagem é um método utilizado pelo enfermeiro para a identificação de alterações no processo saúde-doença, permitindo a prescrição e a implementação de ações de enfermagem que contribuam para a recuperação da saúde e a prevenção de agravos. Além disso, a consulta permite ao profissional de enfermagem atuar de forma direta e independente com o paciente, ao mesmo tempo em que documenta a sua prática $^{(1)}$. Além do mais, permite ao enfermeiro prestar atendimento diferenciado por meio da abordagem personalizada e integral com a finalidade de desenvolver um cuidado direcionado ao indivíduo. Isto possibilita melhoria na qualidade da assistência, pois permite compreender e respeitar o sofrimento vivido pela pessoa com transtorno mental, bem como minimiza o preconceito presente na vida dessas pessoas no decurso da história da saúde mental ${ }^{(6)}$.

Durante a consulta de enfermagem, o enfermeiro deve considerar o paciente como um todo, os aspectos afetados e os sadios, reforçar e estimular as suas potencialidades, a subjetividade do ser cuidado. Deste modo, vínculos entre o profissional e o cliente podem ser construídos a fim de identificar e satisfazer as necessidades deste cliente e obter qualidade no cuidado prestado $^{(6)}$. Assim, a consulta de enfermagem em saúde mental é essencial para que os enfermeiros fortaleçam esta profissão como prática científica ${ }^{(1)}$.

Diante do exposto, o objetivo deste estudo foi descrever os diagnósticos e intervenções de enfermagem resultantes da consulta de enfermagem a pessoas com transtornos mentais e familiares.

\section{MÉTODO}

Pesquisa documental, retrospectiva, de caráter descritivo, a partir dos dados registrados nas fichas utilizadas durante as consultas de enfermagem em Saúde Mental no ano de 2010. As consultas foram realizadas por alunos de enfermagem e enfermeiros em um projeto de extensão desenvolvido no Setor de Ciências da Saúde da Universidade Federal do Paraná na cidade de Curitiba. O referido projeto atende pessoas com transtornos mentais e familiares que, além da consulta de enfermagem, participam de outras atividades, como rodas de conversa, reuniões temáticas e das atividades lúdicas.

A amostra selecionada para a coleta de dados compreendeu todas as 17 fichas de participantes, no total de 44 consultas de enfermagem: 11 de pessoas com 
transtornos mentais e seis de familiares. A diferença no número de fichas para o de participantes se explica pelo fato do Projeto ocorrer por demanda espontânea e, neste caso, cada participante agendou consulta conforme interesse próprio, variando de uma à seis consultas.

A coleta dos dados nas fichas ocorreu entre os meses de fevereiro a julho de 2010, com o uso de dois instrumentos. O primeiro foi direcionado à pessoas com transtornos mentais, contendo informações sobre sexo, idade, situação conjugal, ocupação, diagnóstico de transtorno mental (informado pelo paciente), tempo de tratamento, diagnóstico e intervenção de enfermagem. $\mathrm{O}$ segundo foi direcionado às fichas dos familiares contendo as mesmas informações, acrescidas pelo grau de parentesco. Inicialmente os dados foram organizados em tabelas individuais, em seguida, separados em dois grupos distintos: Grupo P, com dados das pessoas com transtorno mental; e Grupo F, com dados dos familiares.

Os diagnósticos de enfermagem foram identificados de acordo com a taxonomia II, da North American Nursing Diagnosis Association - NANDA ${ }^{(9)}$, e classificados conforme sua estrutura de domínios e classes. Para a padronização das intervenções de enfermagem foi empregada a Classificação de Intervenções de Enfermagem - NIC(10), aplicada em forma de orientação e educação para saúde, sendo apresentada àqueles que tiveram frequência acima de 50\%, em decorrência da quantidade elevada de diagnósticos.

Este estudo faz parte de um projeto maior intitulado "Empowerment dos atores sociais de um espaço de cuidado à saúde de pessoas com transtornos mentais e familiares” aprovado pelo Comitê de Ética em Pesquisa do Setor de Ciências da Saúde da Universidade Federal do Paraná, sob a inscrição CEP/SD: 788.123.09.09; CAAE 0063.0.091.000-09.

\section{RESULTADOS}

Referente aos dados de caracterização, 65\% foi composto pelo Grupo P e 35\% pelo Grupo F. Entre as pessoas com transtorno mental, $55 \%$ eram do sexo masculino e $45 \%$ do feminino, com a média de idade de 53 anos. Concernente ao diagnóstico clínico predominou a esquizofrenia com (45,5\%), seguida da depressão (36,3\%) e transtorno bipolar associado à dependência química $(18,2 \%)$. Quanto à situação conjugal, $9 \%$ eram viúvos, $18,2 \%$ separados $27,3 \%$ casados e $45,5 \%$ solteiros (Quadro 1).

No que se referem aos dados dos familiares, todas são do sexo feminino, com média de idade de 63,5 anos, sendo $83 \%$ delas representadas pela figura materna (Quadro 2).

Na tabela 1 são apresentados os Diagnósticos de Enfermagem referentes ao grupo $P$, com maior frequência para 'interação social e padrão do sono prejudicados', seguidos por 'processo de pensamento perturbado, memória prejudicada, comportamento de saúde propenso a risco e enfrentamento familiar comprometido'.

Quanto aos diagnósticos referentes ao Grupo F (Tabela 2), aqueles que apresentaram maior prevalência foram 'risco para vínculos pais/filhos prejudicados' e 'atividades de recreação deficientes', seguidos por 'desesperança', 'sofrimento moral' e 'nutrição desequilibrada'.

Quadro 1 - Caracterização das pessoas com transtorno mental segundo diagnóstico clínico, sexo, idade, situação conjugal e tempo de tratamento. Curitiba, 2010

\begin{tabular}{|l|l|l|l|l|l|l|}
\hline Participante & \multicolumn{1}{|c|}{ Diagnóstico Clínico } & Sexo & Idade & \multicolumn{1}{|c|}{ Ocupação } & \multicolumn{1}{|c|}{$\begin{array}{c}\text { Situação } \\
\text { Conjugal }\end{array}$} & $\begin{array}{c}\text { Tempo de } \\
\text { tratamento }\end{array}$ \\
\hline P 1 & Esquizofrenia & M & 51 & Aposentado & Solteiro & 30 anos \\
\hline P 2 & Depressão & F & 55 & Aposentado & Solteiro & 17 anos \\
\hline P 3 & Esquizofrenia & M & 32 & Escritor & Solteiro & 12 anos \\
\hline P 4 & Depressão & F & 65 & Babá & Viúvo & 01 ano \\
\hline P 5 & Depressão & F & 55 & Zeladora & Casado & 10 anos \\
\hline P 6 & $\begin{array}{l}\text { Transtorno bipolar; } \\
\text { Dependência química }\end{array}$ & M & 48 & Aposentado & Casado & 16 anos \\
\hline P 7 & $\begin{array}{l}\text { Transtorno bipolar; } \\
\text { Dependência química }\end{array}$ & F & 49 & $\begin{array}{l}\text { Educadora } \\
\text { Social }\end{array}$ & Separado & 26 anos \\
\hline P 8 & Depressão & F & 49 & Comerciante & Casado & 24 anos \\
\hline P 9 & Esquizofrenia & M & 41 & Aposentado & Solteiro & 30 anos \\
\hline P10 & Esquizofrenia & M & 42 & Aposentado & Separado & 25 anos \\
\hline P11 & Esquizofrenia & M & 48 & Aposentado & Solteiro & 31 anos \\
\hline
\end{tabular}


Quadro 2 - Caracterização dos familiares segundo grau de parentesco, idade, situação conjugal, transtorno mental e o tempo de tratamento do familiar adoecido. Curitiba, 2010

\begin{tabular}{|l|l|l|l|l|l|l|}
\hline Participante & Parentesco & Idade & $\begin{array}{l}\text { Situação } \\
\text { conjugal }\end{array}$ & Ocupação & $\begin{array}{c}\text { Transtorno do } \\
\text { familiar adoecido }\end{array}$ & $\begin{array}{l}\text { Tempo de tratamento } \\
\text { do familiar adoecido }\end{array}$ \\
\hline F1 & Mãe & 60 & Separada & Costureira & Esquizofrenia & 30 anos \\
\hline F2 & Mãe & 73 & Viúva & Aposentada & $\begin{array}{l}\text { Esquizoafetivo; } \\
\text { Dependência química }\end{array}$ & 25 anos \\
\hline F3 & Mãe & 73 & Casada & Aposentada & Transtorno bipolar & 10 anos \\
\hline F4 & Mãe & 47 & Casada & $\begin{array}{l}\text { Auxiliar de } \\
\text { Enfermagem }\end{array}$ & Esquizofrenia & Sete anos \\
\hline F5 & Tia & 54 & Casada & Aposentada & Transtorno bipolar & 10 anos \\
\hline F6 & Mãe & 65 & Casada & Comerciante & Dependência química & 42 anos \\
\hline
\end{tabular}

Tabela 1 - Diagnósticos, Intervenções e Resultados de Enfermagem referentes às pessoas com transtorno mental. Curitiba, 2010

\begin{tabular}{|c|c|c|}
\hline Participantes & $\begin{array}{cc}\text { Diagnóstico } & \text { Frequência } \\
\text { NANDA } & (\%)\end{array}$ & Intervenção NIC \\
\hline
\end{tabular}

1. Domínio: Psicossocial. Classe: Papéis/Relacionamento

\begin{tabular}{lr}
\hline P4; P5; P6; & 1. Interação \\
P7; P9; 10; & social \\
P11 & prejudicada \\
\hline
\end{tabular}

P1; P2; P5; 2. Padrão de

P7; P 8; P9; sono

P11 prejudicado
Aumento da socializarão

63,6 1. Encorajar maior envolvimento nas relações já estabelecidas;

2. Encorajar atividades sociais e comunitárias.

\section{Domínio Funcional. Classe: Sono/Repouso}

Melhora do sono

63,6 facilitar a transição do estado de alerta ao estado de sono;

2. Orientar o paciente a evitar ingerir próximo da hora de dormir alimentos e bebidas que interfiram no sono.

3. Domínio Funcional. Classe: Valores e Crença

P4; P6; P8; 3. Sofrimen-

P9; P7; P5 to moral
Melhora do Enfrentamento

54,5 1. Encorajar o paciente a identificar seus pontos fortes e capacidades; 2. Encorajar a verbalização de sentimentos, percepções e medos.

4. Domínio: Fisiológico. Classe: Neurocognição

P2; P3; P5; 4. Memória

P8; P10; P11 prejudicada

P2; P3; P5; 5. Processo P9; P10; P11 dopensamento perturbado
Estimulação cognitiva

54,5 1. Conversar com o paciente / oferecer informações verbais e escritas;

2. Dar orientações sobre tempo, espaço e pessoas.

Estimulação cognitiva

54,5 1. Conversar com o paciente / oferecer informações verbais e escritas; 2. Dar orientações dobre tempo, espaço e pessoas.

5. Domínio Psicossocial. Classe: enfrentamento

\section{Compor-}

P1; P2; P3; tamento de P5; P7; P11 saúde propenso a risco

7. Enfren$\mathrm{P} 4$; $\mathrm{P} 6$; P7; tamento faP8; P9; P10 miliar comprometido Promoção do envolvimento familiar

1. Facilitar a compreensão dos membros da família quanto aos aspectos

54,5 médicos da condição do paciente;

2. Identificar e respeitar os mecanismos de enfrentamento usados pelos membros da família.

Promoção do envolvimento familiar

54,5 médicos da condição do paciente;

2. Identificar e respeitar os mecanismos de enfrentamento usados pelos membros da família. 
Tabela 2 - Diagnósticos, Intervenções e Resultados de Enfermagem referentes aos Familiares. Curitiba, 2010

\begin{tabular}{|c|c|c|c|}
\hline Participantes & $\begin{array}{c}\text { Diagnóstico } \\
\text { NANDA }\end{array}$ & $\begin{array}{c}\text { Frequência } \\
\text { (\%) }\end{array}$ & Intervenção NIC \\
\hline \multicolumn{4}{|c|}{ 1. Domínio Psicossocial; Classe enfrentamento } \\
\hline $\begin{array}{l}\text { F1; F3; F4; } \\
\text { F5; F6 }\end{array}$ & $\begin{array}{l}\text { 1. Enfren- } \\
\text { tamento fa- } \\
\text { miliar com- } \\
\text { prometido }\end{array}$ & 83,3 & $\begin{array}{l}\text { Promoção do envolvimento familiar } \\
\text { 1. Facilitar a compreensão dos membros da família quanto aos aspectos } \\
\text { médicos da condição do paciente; } \\
\text { 2. Identificar e respeitar os mecanismos de enfrentamento usados } \\
\text { pelos membros da família. }\end{array}$ \\
\hline \multicolumn{4}{|c|}{ 2. Domínio Funcional; Classe: Atividade /Exercício } \\
\hline F1; F2; F6; F5 & $\begin{array}{l}\text { 2. Atividades } \\
\text { de recreação } \\
\text { deficientes }\end{array}$ & 66,6 & $\begin{array}{l}\text { Ensino: Atividade/ Exercício prescrito } \\
\text { 1. Informar o paciente sobre o propósito e os benefícios da atividade/ } \\
\text { do exercício prescritos; } \\
\text { 2. Informar o paciente sobre as atividades adequadas, com base na } \\
\text { condição física. }\end{array}$ \\
\hline \multicolumn{4}{|c|}{ 3. Domínio: Psicossocial; Classe: Papéis/Relacionamento } \\
\hline F1; F2; F3; F6 & $\begin{array}{l}\text { 3. Risco } \\
\text { de vínculo } \\
\text { pais/filhos } \\
\text { prejudicado }\end{array}$ & 66,6 & $\begin{array}{l}\text { Promoção da integridade familiar } \\
\text { 1. Estabelecer uma relação de confiança entre os membros da família; } \\
\text { 2. Facilitar a comunicação aberta entre os membros da família. }\end{array}$ \\
\hline \multicolumn{4}{|c|}{ 4. Domínio Funcional; Classe: Nutrição } \\
\hline F1; F2; F5 & $\begin{array}{l}\text { 4. Nutrição } \\
\text { desequili- } \\
\text { brada }\end{array}$ & 50 & $\begin{array}{l}\text { Controle da Nutrição } \\
\text { 1. Encorajar uma ingestão calórica adequada ao tipo de corpo e ao } \\
\text { estilo de vida; } \\
\text { 2. Oferecer informações adequadas sobre as necessidades nutricionais } \\
\text { e a forma de satisfazê-las. }\end{array}$ \\
\hline \multicolumn{4}{|c|}{ 5. Domínio Funcional; Classe: Valores e Crença } \\
\hline F1; F2; F6 & $\begin{array}{l}\text { 5. Sofrimento } \\
\text { moral }\end{array}$ & 50 & $\begin{array}{l}\text { Melhora do Enfrentamento } \\
\text { 1. Encorajar o paciente a identificar seus pontos fortes e capacidades; } \\
\text { 2. Encorajar a verbalização de sentimentos, percepções e medos. }\end{array}$ \\
\hline \multicolumn{4}{|c|}{ 6. Domínio: Psicossocial; Classe: Emocional } \\
\hline F2; F3; F6 & $\begin{array}{l}\text { 6. Desespe- } \\
\text { rança }\end{array}$ & 50 & $\begin{array}{l}\text { Promoção de esperança } \\
\text { 1. Compartilhar os aspectos positivos da esperança; } \\
\text { 2. Informar o paciente sobre o fato de a atual situação ser ou não algo } \\
\text { temporário. }\end{array}$ \\
\hline
\end{tabular}

\section{DISCUSSÃO}

Os dados de caracterização das pessoas com transtornos mentais desta pesquisa vão ao encontro de estudo desenvolvido com usuários de um ambulatório de saúde mental da rede substitutiva ao hospital psiquiátrico da cidade de Natal-RN, ao mostrar que dois terços de pessoas que apresentam algum tipo de transtorno mental são do sexo masculino ${ }^{(11)}$. A presente pesquisa mostra que a esquizofrenia está entre as doenças de maior prevalência, acometendo pacientes com idade média de 30 anos.

Observa-se que os participantes estão em idade produtiva, no entanto, em virtude da doença, eles não conseguem trabalhar. Estudos apontam que os pacientes com algum tipo de transtorno mental têm dificuldades para se inserir no mercado de trabalho formal devido ao seu comportamento decorrente da sintomatologia da doença. Dessa forma, se tornam economicamente dependentes da família que, por sua vez, além dos gastos convencionais, assume os gastos gerados pelo tratamento ${ }^{(2-3,12)}$.

Os dados da situação conjugal do presente estudo corroboram os de pesquisa realizada com usuários de um CAPS da Regiao Sudeste brasileira, que destaca dificuldades de interação pessoal por uma parte sig- 
nificativa desse grupo, principalmente com respeito a relacionamentos amorosos devido aos sintomas gerados pelo transtorno mental ${ }^{(12)}$.

Na caracterização dos familiares, houve predominância da presença da figura materna nas consultas. Assim, compreende-se que a família se torna uma rede de cuidado extra-hospitalar que o indivíduo com transtorno mental possui, destacando a figura do sexo feminino (mães, avós ou irmãs), que se responsabiliza e cuida do membro adoecido ${ }^{(13)}$.

No concernente aos diagnósticos aplicados nas consultas de enfermagem, a interação social prejudicada mostra que, embora as relações interpessoais antes do aparecimento da doença sejam comuns, após o adoecimento elas ficam raras, ou até mesmo inexistentes. Isso ocorre pelas frequentes mudanças de comportamento do indivíduo que resultam, na maioria das vezes, em dificuldades de relacionamento, tanto familiar como social ${ }^{(2,12)}$.

Sabe-se que a participação do paciente em uma rede de atenção extra-hospitalar, como, por exemplo, o CAPS, facilita sua interação interpessoal, promovendo o relacionamento e desenvolvendo os laços afetivos, como também o fortalecimento de vínculos familiares, contribuindo para o sucesso do tratamento ${ }^{(12)}$.

O diagnóstico de enfermagem padrão do sono prejudicado está relacionado aos efeitos adversos das medicações psicoativas. Essas porem alterar os horários e o tempo de sono, fazendo com que o paciente permaneça adormecido além do convencional, como também pode causar a insônia, podendo estar relacionado à dificuldade de relacionamento ${ }^{(2,14)}$.

No que se diz respeito ao processo de pensamento perturbado e memória prejudicada, as pessoas com transtornos mentais, em geral, apresentam algum tipo de deficit cognitivo em diferentes níveis. Esse resulta em capacidade diminuída de atenção, memória e raciocínio lógico, como também, alterações de percepção e de pensamento, originando as alucinações e delírios, respectivamente ${ }^{(15)}$.

O elevado número que pacientes apresentando o diagnóstico comportamento de saúde propenso a risco, é resultado da não adesão ao tratamento. Neste sentido, um estudo realizado com usuários de um ambulatório de saúde mental vinculado ao Centro de Saúde da Faculdade de Medicina de Ribeirão Preto confirma este fato ao mostrar. O estudo mostra que $83,2 \%$ dos pacientes tiverem algum tipo de intercorrências no tratamento, desses, $60 \%$ não fizeram o uso da medicação corretamente, gerando risco para a saúde. Outras intercorrências identificadas foram o absenteísmo às consultas agendadas e o abandono do tratamento. Compreende-se que esses comportamentos podem gerar um agravamento da doença, além de dificultar a reintegração social do paciente, refletindo-se no elevado risco para reinternações ${ }^{(14)}$.

O relacionamento interpessoal entre o paciente e o profissional da área da saúde é uma estratégia facilitadora para um processo terapêutico eficaz, com base na confiança, respeito e liberdade, no intuito de oferecer ao paciente harmonia entre relações sociais e familiares ${ }^{(14)}$. Oferecer atendimento à pessoa com transtorno mental, por meio de orientações em grupos terapêuticos, auxilia o fortalecimento de sentimentos para enfrentar a rotina da doença.

Acredita-se que os enfermeiros não devam se deter somente na prescrição médica e na administração de medicamentos, mas orientar os pacientes para que possam compreender e aceitar a proposta de tratamento, valorizando a importância do uso correto da medicação ${ }^{(12)}$.

As limitações e o sofrimento oriundos do adoecimento, causado pelo transtorno mental, são vivenciados tanto pelo paciente como pelo familiar. Esta realidade se faz presente no diagnóstico de enfermagem enfretamento familiar comprometido, representado em ambos os grupos (familiares, 83,3\%, e pessoas com transtorno mental, 54.5\%). Estes dados vêm ao encontro de achados que afirmam que a família tem um desgaste excessivo no relacionamento em decorrência dos sintomas do transtorno mental, e a presença desses sintomas torna os vínculos frágeis e interfere na qualidade de vida dos envolvidos, causando um desequilíbrio em diversos aspectos do âmbito familiar ${ }^{(2-3)}$.

A família tem suma importância no tratamento da pessoa com transtorno mental, portanto, é necessário que seja incluída no processo de reabilitação do indivíduo. No momento em que o grupo familiar também recebe suporte emocional e orientações dos profissionais acerca da doença, ele passa a compreender e aceitar melhor as dificuldades que enfrenta e cria estratégias para facilitar o convívio com o familiar adoecido ${ }^{(11,16)}$.

Quando um indivíduo adoece, todos os integrantes da família ficam emocionalmente afetados, principalmente os pais que, muitas vezes, se sentem culpados erroneamente pela doença do filho. Quando se trata, especificamente, do transtorno mental, as relações interpessoais mudam, e a família toda se desestrutura ${ }^{(2)}$.

Diante do contexto do adoecimento mental, normalmente apenas um familiar se destaca como cuidador, enquanto os restantes, em geral, não participam do processo terapêutico e se sentem desconfortáveis com 
o comportamento do ente adoecido. Essa conduta pode interferir negativamente, gerando diversos conflitos familiares, o que aumenta as dificuldades de relacionamento, tanto com o cuidador quanto com o paciente ${ }^{(11)}$.

Diante disso, o diagnóstico de enfermagem risco para vínculos pais/filhos prejudicados torna-se mais apropriado para essa situação.

Quanto ao diagnóstico atividade de recreação deficiente, entende-se que o cuidado integral ao indivíduo com transtorno mental, muitas vezes, priva a família de atividades de bem estar e dos compromissos para atender às necessidades do ente adoecido. O cuidador, geralmente, tem dificuldade de distribuir tarefas a outros familiares, exercendo exclusivamente esta função ${ }^{(2,11,13)}$. Nesse sentido, compreende-se que a família se torna exaurida emocionalmente, necessitando encontrar uma maneira equilibrada entre o seu lazer e os cuidados prestados à pessoa com transtorno mental ${ }^{(3)}$.

No que se refere à desesperança e sofrimento moral, de acordo com os achados da literatura, ao saber que um membro da família tem um diagnóstico psiquiátrico de transtorno mental, os sentimento se fragilizam e se manifestam de diferentes maneiras e intensidades em cada um dos familiares que se sentem impotentes diante da situação ${ }^{(2-3,12)}$.

A escuta terapêutica é um instrumento do cuidado, minimiza as angústias e diminui o sofrimento do familiar. Este método é eficiente à medida que a conversa se desenvolve, pois possibilita ao indivíduo ouvir o que está proferindo, induzindo-o a uma autorreflexão. Ao escutar um familiar, seja na linguagem oral ou corporal, o profissional consegue descobrir suas reais necessidades e assim está promovendo o acolhimento e o alívio emocional que antes resguardavam a tristeza o medo e a insegurança ${ }^{(2,11)}$.

Por fim, nutrição desequilibrada é o último diagnóstico dessa amostra. É de suma importância considerar o âmbito emocional, biológico e social de cada familiar, haja vista que a sobrecarga física é o reflexo da sobrecarga emocional. Dessa forma, pode-se entender que o cuidador não está preparado para enfrentar a dinâmica do comportamento da pessoa com transtorno mental ${ }^{(3)}$.

Portanto, a saúde mental e a saúde física do cuidador sofrem consequências por prestar cuidados ao ente adoecido. Entre as consequências mais comuns, podemos citar a alterações do sono, da fadiga, do humor e do apetite, perda e aumento de peso. Destarte, as tarefas devem ser distribuídas entre os demais familiares para que se minimize a sobrecarga produzida pelo desgastante ato de cuidar ${ }^{(3,11)}$.

\section{CONCLUSÕES}

Este estudo possibilitou evidenciar, entre outros aspectos, a relevância da aplicação dos diagnósticos e das intervenções durante a consulta de enfermagem. Este procedimento é respaldado legalmente, exclusivo e, portanto, de competência do enfermeiro, conferindo ao profissional a apropriação de uma prática que concede visibilidade e especificidade ao seu processo de trabalho.

Assim, o diagnóstico e intervenções de enfermagem possibilitam ao enfermeiro uma forma de cuidado ímpar e direcionado, consolidando as ações da enfermagem como um diferencial que oportuniza ao profissional uma prática com qualidade e excelência.

O objetivo deste estudo foi alcançado, tornando possível observar as principais dificuldades enfrentadas por pessoas com transtornos mentais e por familiares, permitindo uma atuação direcionada e apropriada pelos profissionais de enfermagem a essa clientela.

Este estudo possibilitou entender o quanto o transtorno mental interfere no relacionamento interpessoal do contexto familiar e social. Destarte, indubitavelmente, o familiar deve ser inserido no processo terapêutico do indivíduo para obter informações acerca da doença e do tratamento, aprendendo o manejo com o ente adoecido a fim de contribuir para a reestruturação das relações.

\section{REFERÊNCIAS}

1. Mendes MH, Freitas VA, Gomes ET. Consulta de enfermagem: uma prática necessária aos indivíduos com transtornos mentais. Rev Bras Integr. 2009;2(1):225-37.

2. Navarini V, Hirdes A. A família do portador de transtorno mental: identificando recursos adaptativos. Texto Contexto Enferm. 2008;4(17):680-8.

3. Borba LO, Schwartz E, Kantorski LP. A sobrecarga da família que convive com a realidade do transtorno mental. Acta Paul. Enferm. 2008;21(4):588-94.

4. Amarante P. Rumo ao fim dos manicômios. Rev Mente Cérebro. 2006;(164):30-5.

5. Brusamarello T. Cuidado de enfermagem com familiares e pessoas com transtorno mental na extensão universitária [dissertação]. Curitiba (PR): Universidade Federal do Paraná; 2011.

6. Canabrava DS, Vilela JC, Brusamarelo T, Roehrs H, Maftum MA. Consulta de enfermagem em saúde mental sustentada na teoria das relações interpessoais: relato 
de experiência. Cienc. cuid. saude. 2011;10(1):150-6.

7. Horta WA. Processo de enfermagem. São Paulo: EPU; 1979.

8. Canabrava D, Maftum MA. Consulta de enfermagem em saúde mental sustentada na teoria das relações interpessoais de Joyce Travelbee [monografia]. Curitiba (PR): Universidade Federal do Paraná; 2007.

9. Nanda - North American Nursing Diagnosis Association. Diagnósticos de enfermagem: definições e classificações 2007-2008. Porto Alegre: Artes Médicas; 2008.

10. McCloskey JC, Bulechek G. Classificação das intervenções de enfermagem (NIC). $4^{\mathrm{a}}$ ed. Porto Alegre: Artmed; 2008.

11. Severo AKS, Dimentein M. O diagnóstico psiquiátrico e a produção de vida em serviços de saúde mental. Estud. psicol. 2009;14(1):59-67.

12. Moll MF, Saeki T. A vida social de pessoas com diagnósticos de esquizofrenia, usuárias de um centro de atenção psicossocial. Rev. Latino-Am. Enfermagem. 2009;17(6):995-1000.

13. Pegoraro RF, Caldana RHL. Mulheres, loucura e cuidado: a condição da mulher na provisão e demanda por cuidados em saúde mental. Saude soc. 2008;17(2):82-94.

14. Cardoso L, Galera SAF. Doentes mentais e seu perfil de adesão ao tratamento psicofarmacológico. Rev Esc Enferm USP. 2009;43(1):161-7.

15. Almeida EHR. Dignidade, autonomia do paciente e doença mental. Rev. Bioet. 2010;18(2):381-95.

16. Brusamarello T, Guimarães NA, Paes MR, Borba LO, Borille DC, Maftum MA. Cuidado de enfermagem em saúde mental ao paciente internado em hospital psiquiátrico. Cogitare enferm. 2009;14(1):79-84. 